\section{KENTRON}

REVUE PLURIDISCIPLINAIR

DU MONDE ANTIQUE

\section{Kentron}

Revue pluridisciplinaire du monde antique

$25 \mid 2009$

Histoire et actualité des sciences de l'Antiquité

\title{
Les Humanités allemandes en guerre : le Rome et Carthage des antiquisants allemands (1943)
}

Johann Chapoutot

\section{(2) OpenEdition}

1 Journals

\section{Édition électronique}

URL : http://journals.openedition.org/kentron/1491

DOI : 10.4000/kentron. 1491

ISSN : 2264-1459

Éditeur

Presses universitaires de Caen

\section{Édition imprimée}

Date de publication : 31 décembre 2009

Pagination : 77-90

ISBN : 978-2-84133-343-1

ISSN : 0765-0590

\section{Référence électronique}

Johann Chapoutot, « Les Humanités allemandes en guerre : le Rome et Carthage des antiquisants allemands (1943) », Kentron [En ligne], 25 | 2009, mis en ligne le 12 mars 2018, consulté le 17 novembre 2020. URL : http://journals.openedition.org/kentron/1491 ; DOI : https://doi.org/10.4000/ kentron. 1491

\section{(ब) $\Theta \odot$}

Kentron is licensed under a Creative Commons Attribution-NonCommercial-NoDerivatives 3.0 International License. 


\section{LES HUMANITÉS ALLEMANDES EN GUERRE: LE ROME ET CARTHAGE DES ANTIQUISANTS ALLEMANDS (1943)}

Associer les humanités allemandes à la guerre peut surprendre: on voit mal, de prime abord, le rapport qui existe entre de sympathiques savants ou d'innocents philologues et des actions de combat.

Pour entamer la réflexion de manière concrète, parlons d'un livre qui a été publié à Leipzig en 1943, intitulé Rome et Carthage ${ }^{1}$.

On observe qu'au titre est adjoint un sous-titre: «Ein Gemeinschaftswerk». Nous pourrions traduire cela par «un ouvrage en collaboration», qui réunit plusieurs auteurs coordonnés par un directeur de publication. La traduction affaiblirait le terme allemand, qui, littéralement, se traduit par «l'œuvre d'une communauté», et qui n'a pas été choisi au hasard.

Le terme de Gemeinschaft est une notion importante, réitérée, martelée par le discours nazi. Le programme du NSDAP est de faire de l'Allemagne une Gemeinschaft, une communauté organique, naturelle, contre cette Gesellschaft créée par les Lumières et la Révolution française: l'opposition entre ces deux notions est courante en Allemagne depuis le XIX ${ }^{e}$ siècle et est devenue, par l'intermédiaire du sociologue Ferdinand Tönnies, mais aussi de Max Weber, un classique de la sociologie. La Gesellschaft, c'est la société du contrat social, du libre choix, de l'adhésion libre et volontaire; la Gemeinschaft, c'est la communauté de la terre et des morts - pour parler comme Barrès -, du Blut und Boden - pour parler comme Richard Walther Darré $^{2}$-, constituée non par la liberté de ses membres, mais par la nécessité, une nécessité naturelle. La société est un groupe culturel; la communauté, une famille naturelle.

On pourra objecter qu'on est loin, dans notre sous-titre, du débat entre Rousseau et Fichte ou Barrès. Pas tant que cela, si l'on considère que la notion de

1. Cf. Vogt 1943.

2. Richard Walther Darré, agronome et théoricien raciste, est, depuis 1931, le chef de l'Office central de la colonisation et de la race de la SS. Il exerce ensuite les fonctions de ministre de l'Agriculture du Reich. 
communauté a connu une réactivation idéologique au sortir de la Première Guerre mondiale. Après quatre ans de souffrances et d'impôt du sang dans les tranchées, il paraissait inconcevable à bien des anciens combattants que l'on adoptât le contrat social de l'ennemi. Or, c'est précisément ce que fait la constitution adoptée à Weimar le 31 juillet 1919, constitution libérale votée sous les auspices de Gothe et de Schiller, qui fait de l'individu autonome le fondement de la société. Les nazis, comme toute l'extrême droite nationaliste, dénoncent cette importation d'un esprit étranger au Geist allemand et exaltent a posteriori la communauté des tranchées - la Kampfgemeinschaft -, fusionnelle, confusionnelle, communauté idéale, qui répond à toutes les questions posées par la révolution industrielle, l'individualisme, l'exode rural, la dissolution des identités et des solidarités traditionnelles. La tranchée a fondu des atomes épars en un bloc indissoluble, comme le décrit Ernst Jünger, dans Feu et sang, quand il relate la préparation d'artillerie qui précède l'assaut, déluge de feu et d'acier qui s'abat sur l'ennemi:

Devant nous se dresse la plus puissante de nos armes, la muraille de feu et d'acier, haute comme une tour. En ce moment, elle est à notre image: une totalité, mais constituée d'atomes ardents réunis en un seul bloc ${ }^{3}$.

La communauté en guerre, c'est la communauté par excellence: la distinction entre ami et ennemi y est claire, la solidarité spontanée de ses membres y est viscérale et vitale. La communauté, c'est le groupe primaire de la tranchée, emmené par son chef, que tous les anciens combattants, même les pacifistes - comme Remarque et son hymne à Kat dans À l'ouest rien de nouveau -, célèbrent.

À partir de 1933, l'emploi du mot Gemeinschaft connaît une inflation dans le discours nazi. Il est employé à toute occasion pour signifier que " 1789 [devait] être effacé de l'histoire», comme le disait Goebbels, et que l'Allemagne était encore bel et bien en guerre.

L'état de guerre, selon les nazis, se poursuit, car:

- la guerre n'est pas vraiment finie: ni l'armistice, suspension d'armes, de 1918, ni le Diktat ignominieux de 1919 n'ont mis fin à la guerre. Le peuple allemand reste confronté à un monde d'ennemis qui veulent sa perte;

- plus généralement, dans la lutte permanente que constitue le monde humain, dans cet affrontement social-darwiniste des races, la communauté allemande, resserrée, ramassée comme le groupe primaire, est en guerre.

Dès lors, on voit que le sous-titre de l'ouvrage est rien moins qu'innocent, qu'il est, au contraire, lesté de connotations lourdes: que l'ouvrage soit en collaboration, la simple mention du (Hrsg.) - le (dir.) français - aurait suffi à l'indiquer. Introduire

3. Cf. Jünger 1998, 115 . 
une redondance veut dire ceci : il y a une communauté des historiens allemands, et cette communauté est en guerre. Le message sous-jacent est le suivant: les historiens ne sont pas les fieffés individualistes que l'on croit, emmurés dans la solitude de leur cabinet pour déchiffrer de vieux codices, ils travaillent en groupe, ils ont un sens de la communauté - ils ne sont pas de ces insupportables humanistes de la Weimarer Klassik qui pratiquent une ars gratia artis pour leur jouissance propre. Leur activité est rien moins que futile, anodine ou pacifique: ils n'ânonnent pas dates et concepts pour, au mieux, faire passer des examens à des étudiants; ils se battent.

Cette redondance est encore soulignée par le nom de la série dans laquelle est publié l'ouvrage: «Deutsche Geisteswissenschaft». «Sciences humaines allemandes » serait un bon équivalent à ce "Sciences allemandes de l'esprit» fidèle à l'usage germanique - les Allemands opposent depuis Hegel les sciences de la nature aux sciences de l'esprit, une distinction fameuse, qui sera abondamment théorisée par Dilthey et Rickert.

Il y a donc des sciences humaines allemandes, comme il y a aussi, sans doute, des sciences humaines françaises ou britanniques... On songe ici à Joseph de Maistre, dans ses Considérations sur la France (1797): «J'ai vu, dans ma vie, des Français, des Italiens, des Russes, etc.; je sais même, grâce à Montesquieu, qu'on peut être Persan: mais quant à l'homme, je déclare ne l'avoir rencontré de ma vie; s'il existe, c'est bien à mon insu». Il n'y a pas d'homme en soi, pas d'homme universel, il n'y a que des particularismes. Ce qui vaut pour l'homme vaut pour les sciences humaines.

Dire cela, c'est montrer que l'on ne vise pas l'universalité du savoir, mais que tout discours est irréductiblement particulier, situé dans sa nation: il y a une historiographie allemande, qui est, comme la littérature et la philosophie allemandes, l'expression d'un Geist propre à une race. La race s'objective par des créations qui n'appartiennent qu'à elle et dont elle ne se soucie pas qu'elles soient communicables. Comprendra la philosophie allemande tout esprit allemand. Aura accès à la musique et à la littérature allemandes tout esprit allemand. Idem pour l'historiographie. Tout cela restera inaccessible à un Britannique ou à un Russe. C'est que la race n'est pas seulement affaire de substance charnelle et de corporéité: la psychologie raciale a été instituée en discipline et dotée d'un abondant corpus par Ludwig Ferdinand Clauss dans les années vingt et trente ${ }^{4}$.

Dans un contexte de guerre, cette science humaine allemande est une arme de combat: les essais de philosophes et d'historiens, toujours désespérément soucieux de prouver l'utilité de leur discipline - car il en va de leur financement, surtout quand les ressources se trouvent orientées vers ce qui est considéré comme stratégique -, abondent, aux titres explicites: tel professeur disserte sur «l'importance stratégique

4. Cf. Clauss 1926 et 1933. 
de la philosophie ${ }^{5}$, érigée en "arme et instrument» de guerre ${ }^{6}$. L'histoire n'est pas en reste: vecteur d'une "éducation nationale-politique " ${ }^{7}$, elle a son rôle à jouer dans $«$ la guerre des races ${ }^{8}$.

Le livre auquel nous nous intéressons, Rome et Carthage, œuvre de la communauté des historiens allemands en guerre, est ainsi un livre de guerre au double sens du terme: c'est un livre d'histoire, qui parle de l'affrontement pluriséculaire entre Rome et Carthage, et c'est un livre écrit dans un contexte de guerre pour contribuer à la victoire allemande par une mobilisation des esprits allemands. En parlant du passé, ce livre veut contribuer à renforcer la mobilisation culturelle dans cette guerre qui est une guerre totale, entraînant tous les sujets du Reich dans un engagement psychologique total, et dont l'issue, comme le répètent Goebbels et Hitler, sera une victoire absolue, inédite, ou l'annihilation: en 1941 a débuté « une guerre où il n'y aura», dit Goebbels, «ni vainqueurs ni vaincus, mais des survivants et des exterminés ${ }^{9}$.

Comment ce livre contribue à l'édification d'une culture de guerre, nous le verrons plus loin, en parlant de son contenu. Il faut d'abord éclairer plus avant le contexte, cette fois-ci le contexte institutionnel, de son élaboration.

L'éditeur qui publie ce livre est Koehler \& Amelang. Cette maison d'édition est sise à Leipzig, siège d'une grande université fondée en 1409, et dont la faculté d'histoire est réputée. Leipzig est un grand centre d'édition depuis le XIX ${ }^{e}$ siècle, en histoire surtout: il faudra, pour affaiblir ce centre intellectuel, douze ans de nazisme et quarante ans de RDA. Un impressionnant nombre de maisons d'édition y ont leur origine et leur siège, ce qui fait de la ville, avec Munich et Berlin, un des tout premiers centres allemands d'édition, notamment en histoire. Barth, Weicher, Hammer, Hinrichs, Hesse \& Becker s'y trouvent, mais aussi des maisons plus importantes et plus célèbres: outre Ph. Reclam, bien connu en Allemagne pour ses éditions de poche saumon, puis jaune, qui existent depuis la fin du XIX ${ }^{e}$ siècle, B.G. Teubner, W. de Gruyter, Insel, Dürr et Köhler \& Amelang sont présents dans la place, sans compter ces maisons d'édition qui apparaissent après 1933, comme Armanen-Verlag et le Bibliographisches Institut.

Koehler, éditeur prestigieux, crée une nouvelle série, dans laquelle est publié notre ouvrage, qui s'intitule «Deutsche Geisteswissenschaft». Cette série doit accueillir les publications du groupe histoire d'un grand projet multidisciplinaire lancé en 1940, le Kriegseinsatz der deutschen Geisteswissenchaften, que l'on peut

\footnotetext{
5. Cf. Rothacker 1944.

6. Cf. Weinhandl 1940.

7. Cf. Klagges 1937.

8. Cf. Benze 1934.

9. Cf. Goebbels 1943.
} 
traduire par «mobilisation des sciences humaines allemandes». Le mot Kriegseinsatz est en fait plus fort que cela: il signifie engagement guerrier, voire action de guerre - einsetzen est un verbe très présent dans le vocabulaire militaire; il signifie «utiliser un équipement» ou «engager une unité, des hommes». En 1940, les sciences humaines allemandes, pour l'Allemagne, s'engagent donc dans une action de guerre.

Le plan quadriennal de 1936 avait généreusement pensé le financement des «sciences de la nature» et de la matière, celui de la physique et de ses applications techniques, utiles à l'amélioration de l'armement, à la chimie productrice de carburants pour la motorisation de la Wehrmacht et d'ersatz pour pallier les difficultés prévisibles de l'approvisionnement en vivres. Les sciences de l'esprit sont, dans ce contexte de mobilisation de l'économie et de la science, passablement défavorisées.

Les sciences humaines vont s'organiser et réagir, à l'initiative d'un juriste - le droit est une Geisteswissenchaft en Allemagne -, Paul Ritterbusch. Ce professeur de droit public et de droit international, recteur de l'université de Kiel, est un nazi convaincu depuis ses années d'études, lors desquelles il militait déjà. Lors d'une conférence des recteurs qui se tient à Berlin le 11 novembre 1939, et qui a pour thème «Forschung im Kriege» - «la recherche en temps de guerre»-, Paul Ritterbusch propose une action de grande envergure dans le domaine des sciences humaines et sociales. Il contacte dans un premier temps des collègues de sa matière ou de sa région, puis reçoit le soutien du ministère de l'Éducation du Reich, dont le titulaire est d'ailleurs un littéraire, Bernhard Rust, ancien professeur de latin dans l'enseignement secondaire. Ce dernier lui confie en janvier 1940 une mission officielle: diriger un projet intitulé Kriegseinsatz der deutschen Geisteswissenschaften, dont les premières réunions ont lieu en avril 1940. Un an plus tard, déchargé de ses obligations de recteur, Paul Ritterbusch revêt une position dans l'organigramme du ministère et obtient le rang de chef de service. Le projet qu'il a initié et qu'il dirige mobilise, entre 1940 et 1944, cinq cents enseignants-chercheurs dans douze disciplines (sciences de l'Antiquité, anglais, géographie, allemand, histoire - droit et préhistoire -, histoire de l'art, orientalistique, philosophie, romanistique, droit public, droit international, droit civil et droit du travail). Il faut noter que sur ces cinq cents personnes, la moitié a obtenu sa chaire ou sa charge après la prise de pouvoir par les nazis et grâce aux effets de la loi du 7 avril 1933, la loi d'aryanisation de la fonction publique allemande, qui a libéré de nombreux postes. Entre 1941 et 1944 sont publiés dans le cadre de ce projet soixante-sept ouvrages, dont quarante-trois monographies et vingt-quatre collectifs, parmi lesquels deux œuvres en histoire ancienne, Rom und Karthago et Das neue Bild der Antike. Les premières publications sont offertes au Führer le 20 avril 1941, pour son cinquante-deuxième anniversaire. Cette grande opération doit témoigner de la vitalité de la recherche allemande en dépit des 
bouleversements qu'a connus l'université depuis 1933: la science allemande, faite par des aryens, n'a pas besoin des Juifs, chassés de l'enseignement et de la recherche depuis la loi du 14 avril 1933. En outre, le Kriegseinsatz doit montrer aux autorités de l'État et du Parti que les sciences humaines sont utiles à la guerre menée par le Reich. Parallèlement, d'ailleurs, à ce projet au niveau du Reich, et en synergie avec lui, différentes universités organisent des séries de conférences, des séminaires et des travaux censés contribuer à l'effort de guerre allemand. C'est le cas par exemple à Bonn, où la Friedrich-Wilhelm Universität organise une série de "conférences de guerre» (Kriegsvorträge), publiée, dans le cas des historiens de l'Antiquité, en 1944, dans un volume en fort mauvais papier de pénurie, mais très instructif sur la manière dont l'histoire de l'Antiquité est relue et récrite depuis $1933^{10}$. La série éditoriale est intitulée Wissenschaft im Kampf für Deutschland - "La science en guerre pour l'Allemagne».

Les sciences humaines allemandes doivent par ailleurs défendre et illustrer les buts de guerre du Reich, en justifiant, en légitimant, et mobiliser les esprits dans un contexte de guerre totale. Il s'agit de promouvoir scientifiquement, de manière apodictique et incontestable, la vision nazie de l'espace et du temps. La réunion de lancement qui se tient à Kiel les 27 et 28 avril 1940 fixe un Rahmenthema, un cadre général de réflexion et de travail, pour toutes les disciplines: «La mission de cet engagement consiste à élaborer scientifiquement, de manière incontestable, l'idée d'un nouvel ordre européen ${ }^{11}$.

Ce nouvel ordre européen dont le Reich est l'organisateur et la matrice, accouché dans la violence des combats menés par la Wehrmacht et les SS, implique une reconfiguration de l'espace européen sur le principe de la race. Il repose également sur une vision du temps, une réécriture de l'histoire, envisagée désormais sous le jour de la lutte des races et du complot des mauvais contre les meilleurs. Il ne s'agit pas simplement de redessiner l'espace européen, mais de créer les catégories et les concepts, de promouvoir les postulats qui permettent de penser cet espace redessiné: à la reconfiguration topographique doit s'ajouter une nouvelle topique mentale propre à légitimer le projet du Reich en promouvant une vision du monde. Pendant que les économistes consacrent un colloque à la Europäische Grossraumwirtschaft - «l'économie de l'espace grand-européen»-, pendant que les géographes et les juristes dissertent sur ce nouvel espace européen redéfini par le racisme nazi, les historiens convoquent le précédent de l'affrontement entre Rome et Carthage et offrent un double volume censé présenter «la nouvelle image - la nouvelle conception - de l'Antiquité».

10. Cf. Becker \& Chuboda 1944.

11. Cf. Hausmann 1998, 62. 
Le message adressé aux autorités est donc le suivant: les sciences humaines, qui ont une réputation tenace d'inutilité et d'éparpillement individualiste, peuvent et savent s'organiser en communauté de combat quand l'heure sonne. D'où une inflation de vocables militaires: outre le Einsatz, chaque discipline n'est plus un Fach (matière), mais devient une Gruppe ou une Sparte (unité), dirigée par un Spartenleiter ou un Gruppenleiter, un «général» dans cette novlangue nazie, qui remplace dans la SA et la SS le vénérable vocable «General» - allogène, car français - par un roboratif et plus germanique Gruppenführer. Les Gruppenleiter organisent des réunions à Berlin ou à Weimar, dans cet Hotel Elephant où Hitler aime à descendre: la consigne exclut strictement les épouses des enseignants-chercheurs convoqués à ces séminaires. Le Kriegseinsatz est un Männerbund, comme la troupe en guerre, laquelle ne tolère ni divertissement ni amollissement.

Parmi les disciplines engagées dans l'entreprise, il y a les «sciences de l'Antiquité» (Altertumswissenchaften), terme générique qui rassemble l'histoire ancienne et l'archéologie, les sciences auxiliaires, mais aussi les lettres classiques et la philosophie ancienne, puisque à ce groupe disciplinaire collaborent des littéraires et des philosophes. Le groupe est constitué par Helmut Berve, professeur à Leipzig, lors d'une réunion inaugurale et solennelle à l'Université, le 5 octobre 1940. L'invitation qu'il adresse à trois cent cinquante personnes précise les objectifs de l'Einsatz: il s'agit d'exposer et de diffuser des connaissances nouvelles, mais surtout les problématiques nouvelles à propos de sujets "où une conception nouvelle s'est imposée». Berve prévoit de faire rédiger des dictionnaires bilingues grec-allemand et latin-allemand, ainsi que des ouvrages de référence sur l'histoire ancienne, notamment en ce qui concerne la conception antique de l'État, la théorisation et la pratique du sport (Agonistik). La première séance de travail, les 2 et 3 avril 1941 à Berlin-Dahlem, est honorée par la présence du ministre Rust et celle de Rudolf Till, philologue et représentant de l'Ahnenerbe de la SS. Cette réunion élabore le premier grand projet du Kriegseinsatz: éditer un ouvrage intitulé Das neue Bild der Antike, que l'on peut traduire littéralement par «La nouvelle image de l'Antiquité», mais qui signifie quelque chose comme «Pour une nouvelle conception de l'Antiquité ». Il s'agit d'introduire dans l'étude de l'Antiquité le concept angulaire de la révolution idéologique national-socialiste, la race, et la notion de lutte des races comme facteur explicatif de tout devenir historique.

Tout cela est exposé dans le discours que tient Berve, au cours duquel il parle de «révolution de l'esprit» ${ }^{12}$, qui consiste en la "prise de pouvoir national-socialiste», cette «mutation puissante dans l'histoire de notre peuple $[\ldots] »$. Nul doute que «les sciences de l'antiquité, elles aussi, ont été bouleversées par cette révolution de l'esprit».

12. Cf. Hausmann 1998, 130. 
Dans la préface de l'ouvrage, le même Helmut Berve écrit:

L'instinct racial enfin réveillé de notre peuple nous fait reconnaître ces deux peuples de l'Antiquité [les Grecs et les Romains] comme participant de notre race et de notre sang [...]. Notre conscience de race nous les a révélés sous un jour renouvelé et nous les a restitués. C'est sur le fondement de cette foi et de ce savoir que se déroule aujourd'hui le travail de la science allemande ${ }^{13}$.

Tout cela s'accompagne d'une prétention à la scientificité: Berve soutient que ces travaux «satisfont aux sévères exigences de la recherche scientifique, ainsi qu'à ses lois d'airain ${ }^{14}$.

Cet ouvrage comporte deux imposants tomes, de 394 pages sur la Grèce et de 458 pages sur Rome. Il constitue une œuvre de prestige: la liste des contributeurs constitue un Who's who des spécialistes de la Grèce et de Rome. On y trouve même une contribution du jeune Hans Georg Gadamer sur la conception platonicienne de l'État.

$\mathrm{Au}$ total, les textes sont idéologiquement assez anodins. Dans une recension qu'il rédige pour la revue Rasse ${ }^{15}$, un des contributeurs, Fritz Schachermeyr, déplore que sur trente-huit contributions, une seule, celle de Miltner, se fonde réellement sur des postulats raciaux.

Il n'en est pas de même du livre que j'évoquais au seuil de cet article, et qui constitue le second projet du Kriegseinsatz, le collectif publié en 1943 sous la direction du grand spécialiste d'histoire romaine Joseph Vogt, intitulé Rom und Karthago (Rome et Carthage).

Dans cet ouvrage, le préambule de Vogt, intitulé «Notre problématique», est un manifeste programmatique de la nouvelle historiographie allemande, de cette nouvelle histoire de l'Antiquité éclairée par l'idéologie national-socialiste, et qu'il convient désormais d'écrire. L'auteur s'attarde sur le caractère cardinal des concepts de guerre, de race et de guerre des races.

Rome et Carthage, c'est d'abord une guerre à mort, une haine inexpiable, qui résonne étrangement avec les événements contemporains, ou plutôt avec la manière dont les événements contemporains ont été présentés par la propagande nazie. Rome et Carthage sont des noms qui ont depuis longtemps excédé le cadre étroit de leur propre temps pour devenir des archétypes universels, transhistoriques:

Rome et Carthage - voilà deux noms historiques qui sont devenus des concepts, des concepts qui signifient une haine abyssale entre deux peuples et une guerre d'extermination ${ }^{16}$.

\footnotetext{
13. Cf. Berve 1942, 6 (Avant-propos).

14. Ibid.

15. Cf. Schachermeyr 1943.

16. Cf. Vogt 1943, 5 (Unsere Fragestellung).
} 
L'emploi du mot Vernichtungskrieg permet de constater la contamination du discours nazi et l'emploi anachronique de catégories contemporaines transposées à l'Antiquité.

Rome et Carthage sont devenus des concepts, non seulement parce que le caractère inexpiable de leur lutte est devenu exemplaire, mais également parce que, de fait, leur guerre est l'épisode d'un affrontement qui traverse et structure toutes les époques.

En effet, ces deux peuples en lutte sont qualifiés de «rassefremd» ${ }^{17}$, étrangers par la race. Après avoir dévoilé la clef explicative du conflit, Joseph Vogt poursuit en posant une question purement rhétorique:

Pour la recherche contemporaine, une question en vient à occuper le premier plan, qui avait jusqu'alors été peu considérée dans l'examen de ce drame de l'histoire mondiale: ce conflit aux lourdes conséquences a-t-il été déterminé par le patrimoine hérité, par le sang de ces peuples, c'est-à-dire par le fait que le monde de la Carthage punique faisait face à la Rome nordique ${ }^{18}$ ?

Carthage punique, Rome nordique: depuis 1933, l'histoire de l'Antiquité fait l'objet, dans le monde scolaire et universitaire, d'un aggiornamento qui la soumet à une exégèse raciale systématique. Rome est une puissance nordique, car elle a été fondée, comme toutes les grandes civilisations de l'histoire, par des peuples aryens émigrés de leur septentrion originel ${ }^{19}$.

Vogt parle ensuite des sources disponibles en soulignant deux problèmes méthodologiques. Le premier est que, les sources romaines étant incomparablement plus prolixes que les sources carthaginoises, une histoire de vainqueurs est à craindre. Scrupule qui l'honore, quand on constate à quel point l'historiographie est couchée sur le lit de Procuste de l'idéologie.

Le second est que le concept de race, qui vient révolutionner, pour son plus grand bénéfice, il va de soi, l'historiographie, n'était guère familier aux anciens, et que sa mobilisation pour commenter les sources antiques est délicate... En assortissant sa réécriture de réserves méthodologiques, Vogt ne fait que crédibiliser un peu plus l'emploi de cette catégorie nouvelle, déjà imposé par le caractère ronflant des titres de docteur et de professeur alignés par la table des matières. Par l'expression de réserves dont on peine à croire qu'elles soient sincères, il confère à son texte un caractère interrogatif, problématisé et donc d'autant plus scientifique, qui a pour effet de légitimer des affirmations qui viennent soutenir le discours raciste des nazis:

17. Ibid.

18. Ibid., 7 .

19. Cf. Chapoutot 2008. 
Le chercheur contemporain tente de relier les caractères d'un peuple avec telle ou telle composante de sa structure raciale. Il se pose ainsi la question de savoir si, par exemple, la fausseté punique si souvent décriée peut être attribuée à l'élément racial arménoïde, arabique ou hamitique, tous présents dans le peuple punique (Volkstum). Le sol de cette terra incognita scientifique est encore incertain, il exige de l'audace et de la réserve en même temps. Nous avons souhaité accumuler non des suppositions, mais des preuves.

La certitude revient donc vite: l'ouvrage présentera des preuves, et l'on saura à quel élément racial attribuer le mensonge punique - rien n'empêche plus de décrire les guerres puniques comme un affrontement de races.

Les titres des contributions sont éclairants, et leur redondance est explicite. Fritz Schachermeyr propose ainsi une «histoire de Carthage du point de vue de l'histoire raciale» ${ }^{20}$, tandis que Fritz Taeger choisit de disserter sur les «guerres de peuples et guerres de races en Méditerranée occidentale» ${ }^{21}$. Un autre grand historien de la Rome antique, Matthias Gelzer, se penche sur «l'opposition des races comme facteur historique lors du déclenchement des guerres puniques» ${ }^{22}$. Quant à Joseph Vogt, il se réserve de gloser sur «le caractère punique de la dynastie de Septime Sévère ${ }^{23}$.

Le thème choisi par Vogt est d'importance et sert d'avertissement au présent: la dynastie des Sévère a régné au $\mathrm{II}^{\mathrm{e}}$ siècle de notre ère, quatre siècles après la fin des guerres puniques. Or, elle vient d'Afrique et marque la revanche de Carthage: que les empereurs de la ville éternelle soient désormais des Puniques constitue un signe sûr de la dégénérescence raciale et politique de Rome. C'est un topos de la littérature romaine que d'affirmer, à l'instar d'Horace ${ }^{24}$, que «Graecia capta ferum uictorem cepit» et que Rome, vainqueur sur les champs de bataille, a été vaincue par les vaincus.

La vision des guerres puniques que nous présente cet ouvrage s'insère parfaitement dans la réécriture nazie de l'histoire ancienne: les historiens du Kriegseinsatz se font les dociles et fidèles promoteurs d'un canevas présent dans Mein Kampf et développé par Alfred Rosenberg en 1935, dans Le mythe du XXe siècle. Les historiens exemplifient et développent ce que les idéologues ont esquissé.

Rosenberg défend, à l'unisson de son maître et en accord avec des manuels scolaires qui, tous, répètent la même antienne, que Rome a raté sa troisième guerre

20. Cf. Vogt 1943, 9-43 ("Karthago in rassengeschichtlicher Betrachtung").

21. Ibid., 44-82 ("Völker- und Rassenkämpfe im westlichen Mittelmeer").

22. Ibid., 178-202 ("Der Rassengegensatz als geschichtlicher Faktor beim Ausbruch der römisch-karthagischen Kriege").

23. Ibid., 346-366 ("Das Puniertum und die Dynastie des Septimius Severus").

24. Cf. epist. 2, 1, 156 . 
punique. Elle l'a certes gagnée sur le court terme, mais elle l'a perdue à plus longue échéance: les Romains se sont bornés en bons soldats à prendre la ville, à la raser, à tuer sa population masculine et à disperser femmes et enfants. Ils feront de même deux siècles plus tard à Jérusalem, où ils épargneront même les hommes.

Les Romains ont raisonné et agi en stratèges, non en protecteurs responsables de leur peuple, parce qu'ils n'étaient pas éclairés par le concept de race et de lutte des races: dans les guerres puniques, comme auparavant dans les guerres médiques, et comme ensuite dans les guerres juives, l'enjeu biologique excédait de bien loin l'enjeu stratégique; il fallait exterminer, annihiler la population vaincue. Ce n'est pas un ennemi, c'est un foyer de virulence biologique qu'il fallait éteindre et non pousser à sa dispersion hors de son territoire, c'est-à-dire à la diaspora.

L'histoire enseigne que cette erreur fut fatale aux Romains qui, vainqueurs sur le champ de bataille, furent vaincus biologiquement: les ennemis d'hier immigrèrent à Rome, infectèrent le sang nordique romain et détruisirent ce peuple «sûr de lui-même et dominateur ${ }^{25}$.

Rome et Carthage est donc pleinement un livre de guerre. Il parle d'une guerre spécifique, historiquement située, mais qui révèle que, au-delà d'une conjoncture historique précise, il existe un affrontement des races, immémorial, depuis que nordiques et sémites existent.

Ce livre contribue également à la construction d'une culture de guerre. Pendant que les historiens se réunissent, pensent, écrivent et publient dans le cadre de leur Einsatz militairement organisé, supplétif du service des armes - ils réservent d'ailleurs un troisième tome du Das neue Bild der Antike aux camarades mobilisés au front, qui l'écriront à leur retour -, d'autres Einsätze mobilisent des soldats formés à l'idéologie et au discours de l'angoisse eschatologique: les Einsatzkommandos des Einsatzgruppen, sur le front de l'Est, assassinent des centaines de milliers de Juifs et reçoivent des cours du soir, dont les supports, sous forme de fascicules, sont consultables aux archives de Berlin-Lichterfelde. Ces fascicules de formation idéologique sont des précipités, des succédanés de ce que nous venons de voir, de ce que nos historiens écrivent sur l'État grec, sur le Juif à Rome, sur les guerres puniques: tous ces éléments d'une Gleichschaltung, d'un alignement idéologique et d'un aggiornamento de l'histoire ancienne, se retrouvent dans une pédagogie élémentaire et brutale, qui suscite l'angoisse pour suggérer et nourrir la brutalité des actes. La mise au pas des sciences de l'homme, mise au pas sans excès de scrupule et sans réticence, a contribué à celle des intelligences, des consciences et des énergies.

25. Cf. Chapoutot 2008 (Troisième partie). 
Le meurtre nazi, toute l'entreprise génocidaire, se nourrissent de l'angoisse historique et biologique diffusée par ces fascicules, vectorisée par ces cours et, en amont, produite, étayée et promue par nos historiens combattants. Eux qui, intimidés et inquiets de la place prise par les sciences de la matière dans la guerre contemporaine, eux qui voulaient tant se rendre utiles et prouver que leur matière, elle aussi, était d'importance stratégique.

Johann CHApoutot Université Pierre Mendès France - Grenoble II

\section{Références bibliographiques}

Becker O., Chuboda K.F. (1944), Griechenland, Bonn, Universitätsbuchhandlung (Kriegsvorträge der Rheinischen Friedrich-Wilhelms-Universität Bonn; 3).

Benze R. (1934), Geschichte im Rassenkampf, Erfurt, Verlag K. Stenger (Volk und Wissen; 17).

Berve H. (1942), Das Neue Bild der Antike. Bd. I: Hellas; Bd. II : Rom, Leipzig, Köhler \& Amelang, 1942.

Снарочтот J. (2008), Le national-socialisme et l'Antiquité, Paris, PUF (Le noeud gordien).

Clauss L.F. (1926), Rasse und Seele. Eine Einführung in den Sinn der leiblichen Gestalt, Munich, J.F. Lehmann Verlag.

Clauss L.F. (1933), Die nordische Seele. Eine Einführung in die Rassenseelenkunde, Munich, J.F. Lehmann Verlag.

Goebbels J. (1943), Discours du 18 février 1943 au Palais des sports de Berlin, publié dans J. Goebbels, Reden, Bd. II, 1939-1945, H. Helmut (éd.), Dusseldorf, Droste Verlag, 1971.

Hausmann F.R. (1998), «Deutsche Geisteswissenschaft» im zweiten Weltkrieg. Die «Aktion Ritterbusch» (1940-1945), Dresde, Dresden University Press (Schriften zur Wissenschafts- und Universitätsgeschichte; 1 ).

IngRaO C. (2001), Les intellectuels SS du SD, 1900-1945, Thèse de doctorat sous la direction de S. Audouin-Rouzeau et de G. Hirschfeld, Université d'Amiens, 2001, 2 vol., 788 p.; publiée sous le titre Croire et détruire. Les intellectuels dans les services de renseignements de la SS, Paris, Perrin (Synthèses économiques), 2009. 
JüNGER E. (1998), Feu et sang. Bref épisode d'une grande bataille, Paris, Christian Bourgois (traduction par J. Hervier de Feuer und Blut. Ein kleiner Ausschnitt aus einer grossen Schlacht, Magdebourg, Stahlhelm Verlag, 1925).

KLAGGES D. (1937), Geschichtsunterricht als nationalpolitische Erziehung, Francfort-surle-Main, Moritz Diesterweg Verlag.

Losemann V. (1977), Nationalsozialismus und Antike. Studien zur Entwicklung des Faches Alte Geschichte, 1933-1945, Hambourg, Hoffman \& Campe (Historische Perspektiven; 7).

Maistre J. de (1797), Considérations sur la France, Londres.

Rothacker E. (1944), Die Kriegswichtigkeit der Philosophie, Bonn, Universitätsbuchhandlung (Kriegsvorträge der Rheinischen Friedrich-Wilhelms-Universität Bonn - Wissenschaft im Kampf für Deutschland; 37).

SChaChermeyr F. (1943), compte rendu de Vogt 1943 in Rasse, Monatsschrift der Nordischen Bewegung, 10, p. 9-43.

SCHÖNwÄLdER K. (1992), Historiker und Politik. Geschichtswissenschaft im Nationalsozialismus, Francfort-sur-le-Main - New York, Campus (Historische Studien; 9).

Vogt J. (1943), Rom und Karthago. Ein Gemeinschaftswerk, Leipzig, Koehler \& Amelang (Deutsche Geisteswissenschaft), 1943.

Weinhandl F. (1940), Philosophie, Werkzeug und Waffe, Neumünster, Wachholtz.

Wolf U. (1996), Litteris et patriae: das Janusgesicht der Historie, Stuttgart, Franz Steiner Verlag (Frankfurter historische Abhandlungen; 37), 1996. 
